\title{
Ecological Significance of the Interaction of Photosynthesis Light and Dark Processes
}

\author{
V. I. Chikov, G. A. Akhtyamova, L. A. Khamidullina \\ Kazan Institute of Biochemistry and Biophysics, FRC Kazan Scientific Center of RAS, Kazan, Russia \\ Email: vichikov@bk.ru
}

How to cite this paper: Chikov, V.I., Akhtyamova, G.A. and Khamidullina, L.A. (2021) Ecological Significance of the Interaction of Photosynthesis Light and Dark Processes. American Journal of Plant Sciences, 12, 624634.

https://doi.org/10.4236/ajps.2021.124042

Received: January 6, 2021

Accepted: April 22, 2021

Published: April 25, 2021

Copyright (c) 2021 by author(s) and Scientific Research Publishing Inc. This work is licensed under the Creative Commons Attribution International License (CC BY 4.0).

http://creativecommons.org/licenses/by/4.0/

\begin{abstract}
The kinetics of ${ }^{14} \mathrm{C}$ incorporation into glycolate was studied after changing the export of photosynthetic products from the leaf. It has been shown that the ribulose-bisphosphate-oxygenase pathway of glycolate formation works in the stationary state of the plant. An excess of photosyntates or a decrease in the amount of light primary products, as well as nitrates in the leaves, immediately turns on the transketolase pathway of glycolate formation. In this case, part of the oxygen formed in the photochemical reactions of chloroplasts ceases to be released from the leaf. After oxygen receives an electron from ferredoxin in the electron transport chain of chloroplasts, it starts (through photorespiration) the formation of non-carbohydrate photosyntates and metabolic processes in the cytoplasm. It was concluded that the main function of photorespiration in the regulation of photosynthesis is maintaining a balance between light and dark processes of photosynthesis on change of living conditions.
\end{abstract}

\section{Keywords}

Assimilate Transport, Apoplast, Stomata, Invertase, Chloroplast

\section{Introduction}

Recently, UN Secretary General Antonio Guterres announced the possible death of humanity before the end of this century (DIGEST as of October 12, 2020, 11:57) and proposed the global warming action plan, which, he said, threatens the very existence of human civilization. In his opinion, this century may become the last in the history of humankind due to the emerging ecological catastrophe. "If we don't act right now, this century could be the last century for humankind," he warned, speaking to the forum held under the auspices of the international climate initiative TED Countdown. 
What is the reason for this state of our ecology? As we know, all living creatures need oxygen produced in the process of plants photosynthesis. Without oxygen, the biosphere on Earth will cease to exist. Therefore, the main task of humanity is to learn self-regulation of this process in changing conditions and what can be done to preserve life on Earth. It has long been shown [1] that not only oxygen release takes place in plant leaves exposed to light, but also the so-called photorespiration (light-stimulated absorption of oxygen by plant leaves), which reduces total leaf photosynthesis, for many years this process was considered a parasitic process [2]. The investigators searched plant species with low photorespiration and conditions affecting its intensity without much success. But found a new type of photosynthesis (C-4 type), in which photorespiration is hardly noticeable [3] [4].

However, in this direction, nothing significant for reducing photorespiration was no found. The idea of photorespiration parasitism still is asserted to this day. Such is a recent survey work [5], which describes photorespiration as the researchers represented about thirty years ago. Recently, the forty-year work in our laboratory was completed, which clearly showed how leaf photosynthesisis and the whole plant photosynthesisis is endogenously regulated with photorespiration [6] [7].

The quintessence is as follows. It is known from biochemistry that when a $\mathrm{CO}_{2}$ molecule is attached to any organic compound, an acid is formed. In the case of photosynthesis, it is Phosphoglyceric acid (PGA) (in most plant species). Then PGA, reduces to sugars with the use of products from photochemical reactions of chloroplasts (ATP and NADPH), which, most often in the form of sucrose, are exported through phloem vessels from the leaf to other plant organs. But in the extracellular space of the leaf (apoplast) there is an invertase enzyme [Kursanov, 1972], which is adsorbed and acts intra-leaf, on the outer surface of photosynthetic mesophyll cells. In the intercellular spaces of the leaf (apoplast), invertase hydrolyzes sucrose (with the formation of glucose and fructose), thereby hindering its export from the leaf.

But apoplastic invertase is active only in acidic media [8]. Therefore, if, for example, light exposure of the leaf has decreased and minor "assimilation power" (ATP and NADPH) is formed in chloroplasts, then the amount of PGA reduced to sugars decreases. This means that the remaining acids cannot be recovered and exported from the leaf as sucrose. Organic acids begin to accumulate in chloroplasts. Excess acids are carried out into the cytoplasm, where they accumulate in vacuole. But photosynthesis is a very intense metabolite flow and vacuole cannot fit them all. A leaf that has completed its growth can use max 5\% - 7\% of its photosynthetic products for metabolism [9]. There is no way out for the rest. Therefore, acids go to the extracellular space, where they activate invertase by increasing the medium acidity.

Glucose and fructose formed during the sucrose hydrolysis increase sugar concentration in the aqueous medium of the leaf apoplast (since two hexose molecules are formed instead of one sucrose molecule). This increases osmolality of 
aquatic medium around the stomata, which degrades the guard cells turgor and they close. The $\mathrm{CO}_{2}$ transport into the leaf through stomata is reduced, and the resulting (with a decrease in illumination) deficiency of "assimilation power" complies with the amount of organic acids already formed. The steady-state comes and everything returns to normal.

In increase of the leaf light exposure, the opposite process will take place. All organic acids formed will be completely recovered and exported from the leaf as sucrose. Acidity of the extracellular space will decrease. Invertase is deactivated and intra-leaf osmosis of the aqueous medium will decrease. The stomata will open and $\mathrm{CO}_{2}$ inflow into the leaf will increase photosynthesis. Light and dark processes of photosynthesis are well-balanced.

But glycolate metabolism (the main participant in photorespiration) is also involved in the formation of organic acids. Moreover, this metabolism pans the entire cell, and not only the chloroplast [2] [10] and is involved in photosynthesis regulation of the whole leaf and the whole plant. Moreover, these processes are proportionate in power and speed of response to changes in photosynthesis conditions. In a series of works back in the 70s of the past century, it was shown [11] [12] [13] [14] that when plant organs consuming photosynthetic products (flowers, fruit elements, apical points) are removed, photorespiration increases (the Warburg effect: an increase in the photosynthesis intensity during decrease in oxygen concentration from $21 \%$ to $1 \%$ ), and total photosynthesis decreases.

Changes in gas exchange upon inhibition of leaf export function are always accompanied by the resulting ${ }^{14} \mathrm{C}$-labeled photosynthetic products ratio distortion [14]. It has long been known [15] that any adverse impact reducing photosynthesis always reduces the sucrose synthesis and enhances its non-carbohydrate direction. That means, less sugars are formed and relatively more organic and amino acids are synthesized. More proteins are formed as the end products of photosynthesis.

The key regulatory compound in photorespiration is glycolic acid, which is formed from ribulose biphosphate (RBP), the first compound that binds $\mathrm{CO}_{2}$ in photosynthesis. This happens due to more successful competition of $\mathrm{O}_{2}$ (as compared to $\mathrm{CO}_{2}$ ) for active centre of princicple enzyme of photosynthesis, ribulose biphosphate carboxylase/oxygenase (RBPC/O). The point is that the RBPC/O enzyme has a dual function. If oxygen enters the active centre of RBPC/O, then as a result of (oxygenation reaction) one glycolate molecule and one phosphoglyceric acid (PGA) are formed. And if $\mathrm{CO}_{2}$ gets to the active centre of $\mathrm{RBPC} / \mathrm{O}$, then (in carboxylation reaction) two PGA molecules are formed [2].

PGA then, as already mentioned, reduce to sugars due to NADPH and ATP formed in the photochemical processes of photosynthesis. And then carbon (already in the form of sucrose) is exported from the leaf to organs that consume photosynthesis products. Glycolate, by participating in photorespiration (with the release of $\mathrm{CO}_{2}$ ), forms amino acids used in protein synthesis. Thus, if RBPC/O binds carbon dioxide, sucrose is formed, which is the main export product of photosynthesis. And if oxygen, then amino acids are formed, substrates for proteins 
synthesis in leaf cells. These acids increase the non-carbohydrate component of photosynthesis. And primarily glycolate, glycine, serine. It should be noted that various mechanisms in the photochemical stage of photosynthesis were created by nature mainly to protect the photosynthetic apparatus of a plant from photodegradation [16] and are not directly related to regulation of plants' production process.

As shown [2] [17], in photosynthesis, glycolate can be formed not only through $\mathrm{RBPC} / \mathrm{O}$, but also through the transketolase reaction (TKR). This enzyme, in the photosynthetic Calvin cycle, transports two-carbon fragments from one sugar phosphate to another, so that a $\mathrm{CO}_{2}$ acceptor (RBP) is formed. But this reaction requires oxygen, which should be activated by receiving an electron from ferredoxin in the electron transport chain of chloroplasts (ETCC). To learn the role of each of these mechanisms in the regulation of photosynthesis and photorespiration, kinetic studies of incorporation of ${ }^{14} \mathrm{C}$ into glycolate, after impairment of export function in experimental leaf, were carried out.

\section{Methods}

The experiments were carried out in Central Asia on cotton plants grown in the field according to standard agricultural techniques. Assimilate excess was made by removing all fruit elements (about 20 - 30) (balls, flowers) from the plant. The removal was carried out in the evening of the day preceding the experiment, and the study of photosynthetic metabolism of ${ }^{14} \mathrm{CO}_{2}$ on next afternoon (12-15p.m.)

The study of the primary $14 \mathrm{C}$ photosynthesis products formation was carried out kinetically on leaves not separated from the plant in daylight. For this, leaves were selected with increasing exposures in photosynthetic chamber with ${ }^{14} \mathrm{CO}_{2}$ $(10,20,40,60,120$ and 180 s). Carbon dioxide concentration maintained at the same level only when experimental leaf was in the photosynthetic chamber and at all exposures was either 0.03 or 0.3 vol.\%. One fixed sample was initially one leaf not separated from one experimental plant (control or experimental). The same was performed in six replications. Leaves fixation after exposure to ${ }^{14} \mathrm{CO}_{2}$ was carried out with boiling ethanol, and fixed samples processing was similar to that described [18].

\section{Results and Discussions}

Let us consider the features of the kinetics of ${ }^{14} \mathrm{C}$ entry into glycolate at a natural carbon dioxide concentration of $0.03 \%$ (Figure $1(\mathrm{a})$ ). In the leaves of experimental plants, radioactivity of glycolate (\% of the total radioactivity of the entire leaf) was maximum at the shortest leaf exposure in the leaf chamber (10 s). Then it rapidly decreased as the leaf exposure time increased in ${ }^{14} \mathrm{CO}_{2}$. That was due to metabolization of ${ }^{14} \mathrm{C}$-PGA and accumulation of pools of ${ }^{14} \mathrm{C}$-labeled products of photosynthesis in subsequent compounds (up to the final product of photosynthesis-sucrose). That showed that in leaves with excess assimilates, glycolate is formed together with the very first ${ }^{14} \mathrm{C}$-labeled substances. 


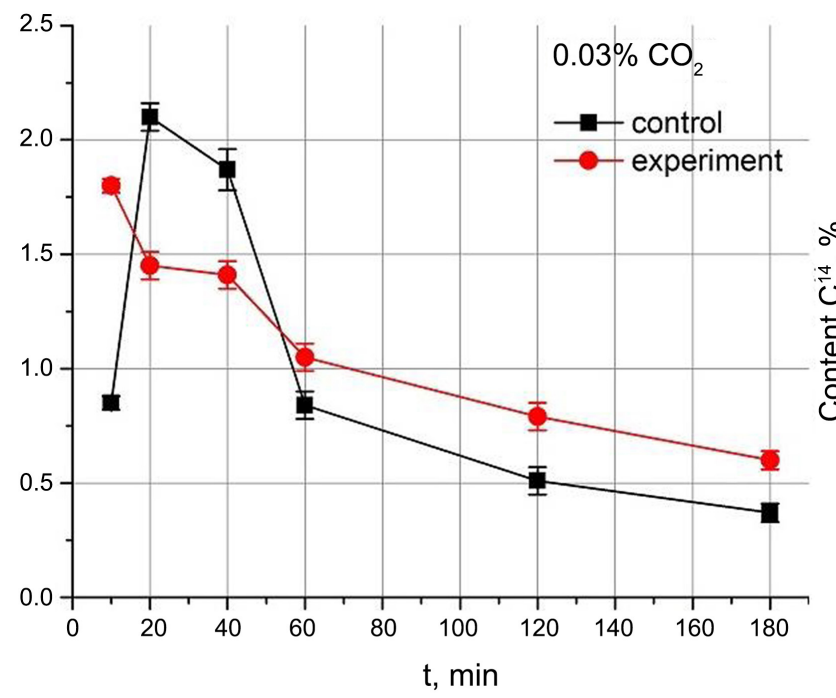

(a)

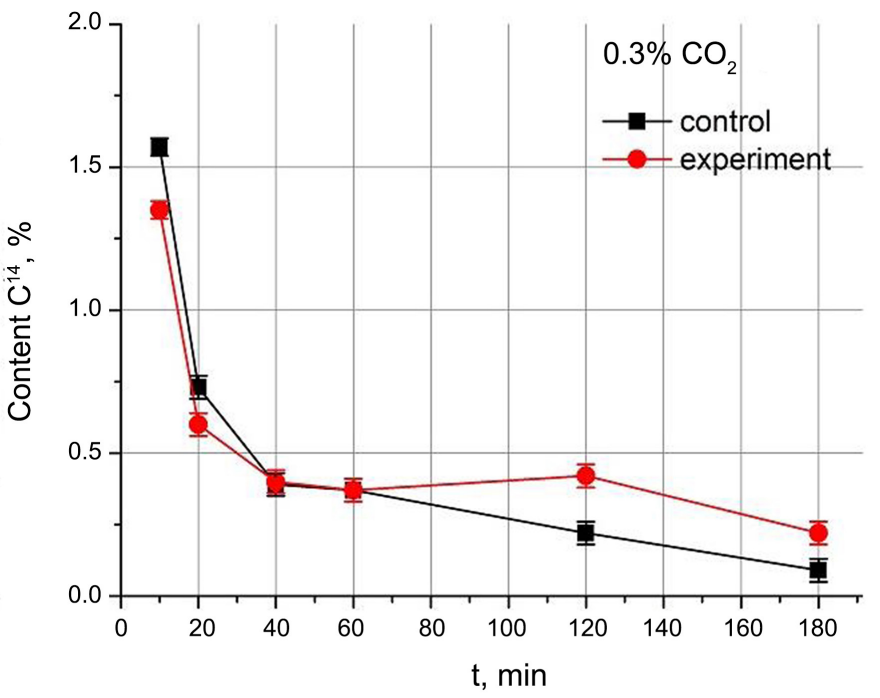

(b)

Figure 1. Effect of fruit elements removal (control and experiment) on photosynthetic incorporation of ${ }^{14} \mathrm{C}$ from ${ }^{14} \mathrm{CO}_{2}$ into glycolate in cotton leaves. (\% of the of the water-ethanol soluble fraction radioactivity). Notation: (a) $\mathrm{CO}_{2}$ concentration $(0.03 \%)$ and (b) $\mathrm{CO}_{2}(0.3 \%)$.

Control plants, unlike experimental ones, showed very small radioactivity in the glycolate in the first seconds (Figure 1(a)). But with an increase in the leaf exposure time at ${ }^{14} \mathrm{CO}_{2}$ (up to 20 and $40 \mathrm{~s}$ ), it increased and exceeded the values of experimental leaves after a $10 \mathrm{~s}$ leaf exposure, and then rapidly decreased to the steady-state level, which after a $180 \mathrm{~s}$ exposure was almost two times lower than in plants with removed fruit elements. Thus, the kinetic curve of ${ }^{14} \mathrm{C}$ inclusion in glycolate in the steady-state (control plants) twice crossed the similar curve in the graph for plants with excess assimilate leaves (Figure 1(a)).

At a saturating concentration of carbon dioxide (0.3\%), kinetic differences (between control plants and without fruit elements) in the label transport into glycolate were not observed. However, there was a one-time (first) intersection of the curves. The great similarity of the kinetics of ${ }^{14} \mathrm{C}$ transport into glycolate in experimental plants at both concentrations of carbon dioxide indicates that glycolate formation in these plants is mainly carried out by a mechanism which does not involve the RBP-oxygenase reaction.

What is the reason for these differences in the ${ }^{14} \mathrm{C}$ inclusion in glycolate? In fruit-free plants, sugar export from leaves almost stopped, photosynthesis is reduced [2], since the removal of such a number of assimilate acceptor organs causes an extraordinary excess of photosynthetic products in the leaf. That means that the use of "restorative power" in chloroplasts (the conditions: clear day, temperature above $+35^{\circ} \mathrm{C}$ ) is difficult. Oxygen generated from water fills the entire leaf.

Under the said conditions, when electrons transport in ETCC from ferredoxin to $\mathrm{NADP}^{+}$is difficult due to its lack in the oxidized state, then electrons in ETCC are transported to $\mathrm{O}_{2}$ with the formation of a superoxide radical $\left(\mathrm{O}_{2}^{-}\right)$. Superoxide radical, oxidizing two-carbon fragments in the transketolase reactions of the 
Calvin cycle, forms glycolate. Thus, the flow of assimilates to export sugars is interrupted, and it is directed to intracellular metabolism.

The essence and difference of these two concepts of glycolate formation is shown by diagram in Figure 2. It clearly shows how the ${ }^{14} \mathrm{C}$ pathway from ${ }^{14} \mathrm{CO}_{2}$ to glycolate formation via TKR is much shorter (only two reactions) compared to the oxygenase reaction, requiring $7-8$ reaction steps to reach the labeled ${ }^{14} \mathrm{C}$-RBP.

And after carboxylation of ${ }^{14} \mathrm{C}-\mathrm{RBP}$ and up to ${ }^{14} \mathrm{C}$-PGA (phosphoglyceric acid). In this case, we can see that in the result of carboxylation of ${ }^{14} \mathrm{C}-\mathrm{RBP}$, two labeled ${ }^{14} \mathrm{C}$-PGA molecules are formed, which is reflected in the kinetics of ${ }^{14} \mathrm{C}$ inclusion into glycolate (Figure 1).

The inhibition of sugars export from leaf also results in the decrease in released inorganic phosphate (after carbon export from leaf in the form of sucrose) return to chloroplasts from cytoplasm. Without phosphate, recovery of PGA in the Calvin cycle, and then its metabolism to sugars, is impossible. Therefore, the consumption of reducing agent $\mathrm{NADPH}^{+}$for the same purposes is also reduced. This automatically hinders electrons transport to photosynthetic electron transport chain to $\mathrm{NADP}^{+}$-oxidized (lacking), and increases the possibility of ferredoxin reducing free oxygen (Mehler reaction) and superoxide radical formation. This radical enhances glycolate formation in chloroplasts through the transketolase reaction.

It is also important that glycolate formed upon activation of photorespiration metabolizes in the cytoplasm to glycine, which further in the reaction:

2 glycine $\rightarrow$ serine $+\mathrm{NADPH}++\mathrm{CO}_{2}$ is converted to serine.

As a result, a reducing agent appears in the cytoplasm to provide synthetic processes already inside the cell. An additional energy resource (ATP) is also

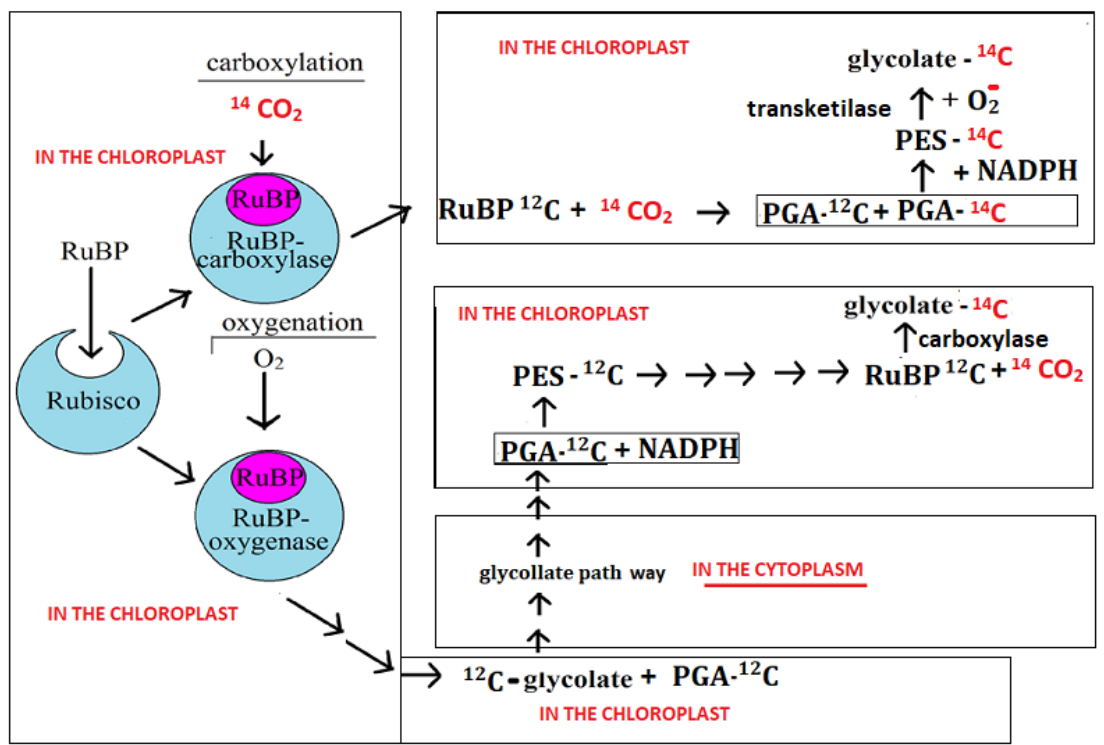

Figure 2. Difference in the ways of glycolate formation in leaf mesophyll cells through oxygenase or transketolase reactions. 


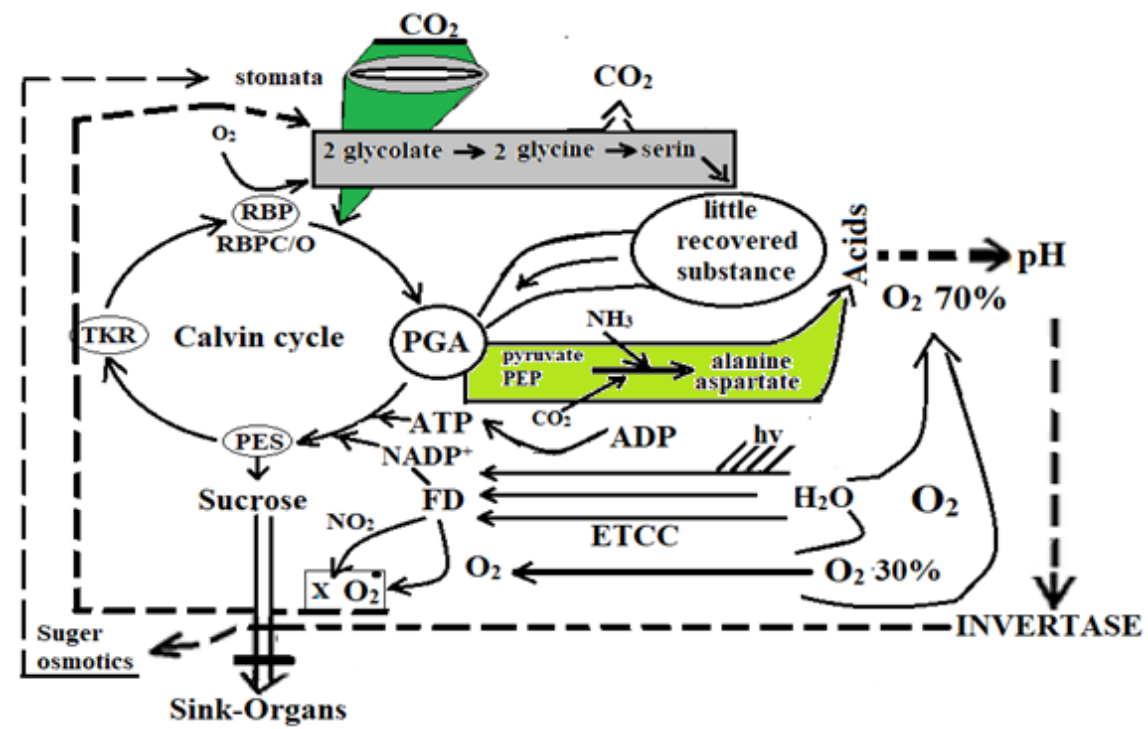

Figure 3. Scheme of carbon photosynthetic metabolism regulation with changes in intensity of assimilates export from the leaf. The dotted line indicates processes that are activated when sugars export from the leaf is inhibited, and the cross line shows inhibited processes. Notation: ETCC-photosynthetic electron transport chain of chloroplasts, FDferredoxin, RBP - ribulose-1, 6-diphosphate, PGA-phosphoglyceric acid, PES-phosphorric esters of sugars, TKR-transketolase reaction.

formed in pseudocyclic photophosphorylation in ETCC when the Mehler reaction is enhanced. That means that both substrate an energy supply of metabolism increase in the cell. Leaf cells can resume growth, even if ontogenetically the leaf has already completed its growth. In this case, the leaf cells growth is resumed not through cell division, but by stretching them to accommodate the excess of photosynthetic products. All these events are graphically presented in the diagram (Figure 3).

In the course of field experiments on cotton in Central Asia, we repeatedly observed how, a few days after the held experiments, the leaves on plants with removed fruit elements increased their size by $2-3$ times.

\section{Conclusions}

Similar changes in metabolism also take place in the leaf in case of mineral fertilizers (primarily nitrates) concentration increase in soil [18]. When $\mathrm{NO}_{2}^{-}$is reduced in ETCC, the radical " $\mathrm{X}$ " is simultaneously formed, which is involved in glycolate formation in the TKR (Figure 3). Nitrates also inhibit the sucrose export from leaves (by activation of invertase with excess organic and amino acids), reduce the ratio of labeled sucrose/hexose and stimulate the growth of mesophyll cells [18]. It is important that in nitrate plants, the inclusion of glycolate pathway in the products increases by 2 - 3 times. It happens even regardless of changes in illumination or $\mathrm{CO}_{2}$ concentration. This is due to the active formation of radical "X". At the same time, final intensity of photosynthesis in nitrate plants (due to the diversion of the carbon flux into photorespiration) is signifi- 
cantly lower [18]. Thus, the activation of transketolase pathway of glycolate formation is the main regulatory mechanism in the light and dark processes ratio distortion in photosynthesis. If sugars export from the leaf is hindered, then the unused flux of quanta results in photodestruction of chloroplasts [19]. And it is a very important factor, since plant does not use the light energy for photosynthates production by more than $1 \%$. The rest energy is dissipated and converted into heat.

That means that nature has found the only possible way to regulate photosynthesis in changing conditions (primarily high-illumination level) through photorespiration, and then through change in the acidity of the aqueous medium in the apoplast. In this case, the start and restructuring of photorespiration occurs at the very initial stage of photosynthesis light and dark processes interaction. In addition, photorespiration encompasses the cell metabolism, since glycolate metabolism passes from chloroplasts into cytoplasm, and part of its metabolism also occurs in mitochondria. Moreover, not only the number of amino acids for proteins synthesis expands, but also the respiratory energy supply of the entire mesophyll cell is maintained. Consequently, with any change in the conditions of plant existence and the intensity of carbon flux through photosynthesis, the orientation of photosynthetic carbon metabolism changes and is regulated through the transketolase reaction. That influences oxygen content in the atmosphere.

The intensity of photorespiration process usually ranges from $25 \%$ to $50 \%$ of the stationary photosynthesis level [20]. Therefore, this is a very important process for the Earth's ecology. Oxygen reduction even by $10 \%$ is too much. It is especially important for ecology that in the course of glycolate metabolism, not only the oxygen content in the atmosphere decreases, but also the carbon dioxide concentration increases, which means the increase of greenhouse effect in the biosphere. That is the major concern of ecologists for a long time.

Presently the concentration of carbon dioxide in the air is $0.05 \%$. But quite recently it was $0.03 \%$. Wherein animals (and humans) exhale $4 \%$ of carbon dioxide. The difference in $\mathrm{CO}_{2}$ concentration between inhalation and exhalation is 80 times. It's very comfortable to breath. But let's imagine that concentration of carbon dioxide will increase to only $2 \%$. The respiratory biochemical reaction of decarboxylation (removal of $\mathrm{CO}_{2}$ from organic molecule) will be inhibited. And the animals will start suffocating. It is especially important for marine animals, since oxygen is hydrophobe, and carbon dioxide is highly soluble in water. Therefore, the ecological catastrophe will begin with the death of marine animals, which will be followed by death of land animals (by $\mathrm{CO}_{2}$ flow into the atmosphere). The possibility of such an outcome depends on accumulation of animals corpses in ocean trenches. We will hope that we did not cross the point of no return.

Besides, in the dark, the $2 \%$ concentration of $\mathrm{CO}_{2}$ depresses dark respiration even for plants. And after such darkness, oxygen production by plants even in the light may be reduced. Difficulties in assimilates export from leaves is of particular importance for woody plants. The recent years growth of massive forest 
fires around the world is also due to a slowdown in assimilates export from leaves and root mass decrease in relation to leaf mass. Nitrates on leaves (carried with the wind as dust from agricultural fields) have a much stronger negative effect than nitrates in the soil.

Once we carried out experiments on cotton in the Yavan Valley in Tajikistan in June and observed such a picture. On a clear day, no sun could be seen in the sky, and there was such a layer of dust on cotton leaves that we could not conduct experiments. As the locals told me, it was the Afghan wind-the wind blowing from south to the north. Naturally, cold air masses descend from the pole to the equator. There they heat up and, taking dust and fertilizers from fields with the wind, rise, carrying them back to the pole. On the way, this dust accumulates on leaves of trees.

Nitrates immediately activate protein metabolism and leaf growth. As a result, the root/leaf mass ratio drops dramatically. The excessive use of mineral fertilizers in agriculture has been going on for over 100 years. That is the reason of few roots in wood trees. And no one thinks of that. The roots of trees cannot supply plant with water. With the slightest decrease in water supply, plants become especially fire-vulnerable. Under such conditions, water flows into the tree crown only through living tissues near the tree bark. The middle part of tree trunk (dead xylem) dries up. This can be clearly seen in the presented (Photo 1) of a tree from Australia. There is a burning tree. It has almost burned out. But it burns inside, where there is dry and dead wood. While tissues adjacent to the bark do not burn. Apparently, they still retain some moisture coming from the soil, even at the end of the whole tree burning. That is the main reason for the said forest fires.

What's the way out? Look for ways to enhance the export function of plant leaf. For this, it is necessary to stop using mineral fertilizers in agriculture [21]; https://regnum.ru/news/innovatio/2374286.html?t=1517388452), which consumes half of energy resources of mankind. Increase plants symbiosis with microorganisms,

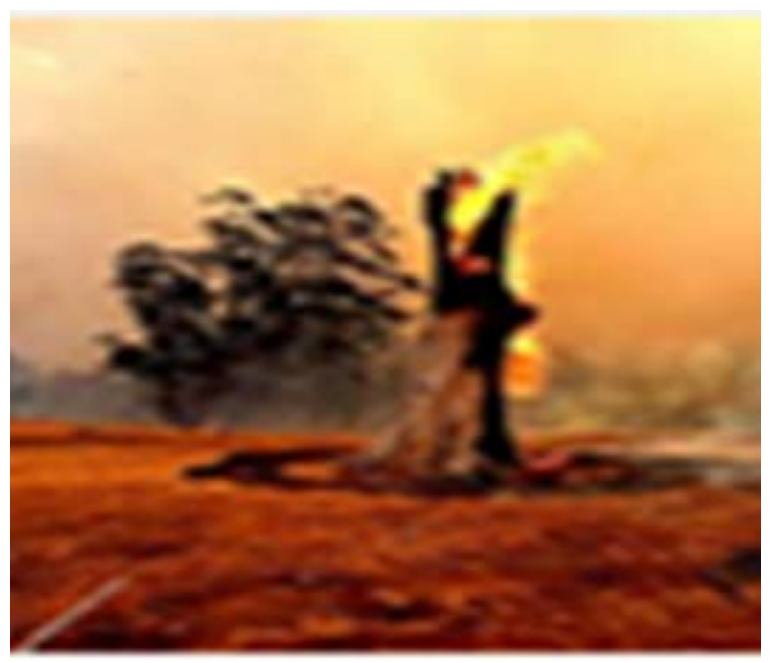

Photo 1. Forest fires. 
which will supply plant with mineral nutrients from soil and air (nitrogen). Our two-meter black soil was formed without mineral fertilizers. It is necessary to increase assimilates export from leaves to roots.

To enhance export of assimilates from plant leaves, we propose [22] [23] complex compounds of copper and zinc with ammonia (ammoniates), which, at a concentration of $10^{-4}-10^{-5} \mathrm{M}$, alkalinize (after spraying tree crowns in the early morning) extracellular fluid of leaves, inhibit invertase. As a result, open stomata provide increased photosynthesis, and most importantly, sugars export from leaves to roots is not inhibited.

\section{Conflicts of Interest}

The authors declare no conflicts of interest regarding the publication of this paper.

\section{References}

[1] Gibbs, M. (1972) Inhibition of Photosynthesis by Oxigen and Glicolic Acid Sinthesis. In: Theoretical Basis of Photosynthesis Productivity, Nauka, Moscow, 205.

[2] Chikov, V.I. (1996) Photorespiration. Educate Journal, No. 11, 2-8.

[3] Karpilov, Y.S. (1960) Distribution of Radioactive Carbon 14C among the Products of Corn Photosynthesis. Proceedings of the Kazan Agricultural Institute, Vol. 41, 5-13.

[4] Hatch, M.D. and Slack, S.R. (1966) Photosynthesis by Sugar-Cane Leaves: A New Carboxylation Reaction and the Pathway of Sugar Farmation. Biochemical Journal, 101, 103-111. https://doi.org/10.1042/bj1010103

[5] Rakhmankulova, Z.P. (2018) Photorespiration: Role in the Production Process and in the Evolution of C4 Plants. Russian Journal of Plant Physiology, 65, 163-179. https://doi.org/10.1134/S1021443718030068

[6] Chikov, V.I., Akhtiamova, G.A., et al. (2015) Effect of Silencing of the Apoplastic Invertase Gene on Photosynthesis in Tomato. Russian Journal of Plant Physiology, 62, 39-44. https://doi.org/10.1134/S1021443715010045

[7] Chikov, V.I. (2017) The Participation of Apoplast Invertase in the Regulation of Photosynthesis by Stomatal of Mechanism. Journal of Plant Sciences, 5, 134-145.

[8] Kursanov, A.L. (1984) Endogenous Regulation of Transport of Assimilates and Sink-Source Relations in Plants. Soviet Plant Physiology, 31, 579-595.

[9] Keerberg, O.F. (1972) Effect of Light Intensity on the Products of of Photosynthesis in Bean Leaves. In: Theoretical Foundations of Photosynthesis Productivity, Nauka, Moscow, 200.

[10] Chikov, V.I., Akhtyamova, G.A. and Khamidullina, L. (2019) The Participation of Photorespiration in the Regulation of Photosynthesis. International Journal of Recent Scientific Research, 10, 30693-30696. https://doi.org/10.24327/IJRSR

[11] Hall, A. and Brady, C.J. (1977) Assimilate Source-Sink Relationships in Capsicum annuum L. II: Effects of Fruiting and Defloration on the Photosynthetic Capacity and Senescence of the Leaves. Journal of Plant Physiology, 5, 771-779. https://doi.org/10.1071/PP9770771

[12] Lenz, F. (1977) Einflu $\beta$ der Frucht auf Photosynthese und Atmung Ztschr. Pflanzenenernahr. und Bodenk. Bd. 140, 51-61. https://doi.org/10.1002/jpln.19771400107 
[13] Chikov, V.I. and Isfandiyarov, N.I. (1978) Effects of the Rate of Assimilate Efflux on the Ratio between Some Components of Plant Gas Exchange. In: All-Union Conference Biochemical and Biophysical Mechanisms of Substance Transport in Plants and Its Regulation, USSA, Gorky, 182.

[14] Chikov, V.I. (1987) Photosynthesis and Transport of Assimilates. Science, Moscow, $188 \mathrm{p}$.

[15] Tarchevsky, I.A. (1965) On the Relationship between the Photosynthetic Phosphorylation Assimilates of $\mathrm{CO}_{2}$ and Other Function of Chloroplasts and Photosynthetic Cells. In: Biochemistry and Biophysics of Photosynthesis, Nauka, Moscow, 305-319.

[16] Karapetyan, N.V. (2008) Protective Dissipation of Excess Absorbed Energy by Photosynthetic Apparatus of Cyanobacteria: Role of Antenna Terminal Emitters. Photosynthesis Research, 97, 195-204. https://doi.org/10.1007/s11120-008-9336-8

[17] Asami, S. and Akasawa, T. (1975) Biosynthesis Mechanism of Glycolate in Chromation II: Enzymatic Mechanism of Glycolate Formation by Transketolase System. Plant and Cell Physiology, 16, 805-814.

[18] Chikov, V.I., Bakirova, G.G. and Ivanova, N.P. (1998) The Role of Photo-Oxidation Processes in Carbon and Nitrogen Metabolism at Photosynthesis of the Wheat Flag. Physiology and Biochemistry of Cultivated Plants, 30, 323-332.

[19] Shabashvili, E.Z. and Chikov, V.I. (1992) Structural and Functional Response of Chloroplasts of Cotton Leaves to Change the Donor Acceptor Relationship in the Plant. Soviet Plant Physiology, 39, 480-485.

[20] Chikov, V.I., Yargunov, V.G., Fedoseeva, E.Z. and Chemikosova, S.B. (1982) Effects of Correlation between Assimilate Production and Usage on Functioning of the Photosynthetic Apparatus in Plants. Soviet Plant Physiology, 29, 1141-1146.

[21] Chikov, V.I. and Akhtyamova, G.A. (2018) Breeding, Genetics and Evolution of Plants: Metabolic Link. Genetics and Bioengineering, 4, 13-19.

[22] Chikov, V.I. (2002) The European Bureau of the World Intellectual Property Organization. N WO 02/092538 A2. 21.11.

[23] Chikov, V.I., Bakirova, G.G., Batasheva, S.N., et al. (2006) The Influence of Ammoniates on ${ }^{14} \mathrm{CO}_{2}$ Assimilation in Flax. Biologia Plantarum, 50, 749-751.

https://doi.org/10.1007/s10535-006-0122-1 